\title{
Intra- Local Toxicology Aspect Time Related in Some Pathologic Conditions
}

\author{
Luisetto Mauro* \\ Europeans Specialist Lab Medicine, Italy
}

Submission: December 01, 2017; Published: December 22, 2017

*Corresponding author: Luisetto M, Applied pharmacologist, Europeans specialist lab medicine, Italy 29121 Email: maurolu65@gmail.com

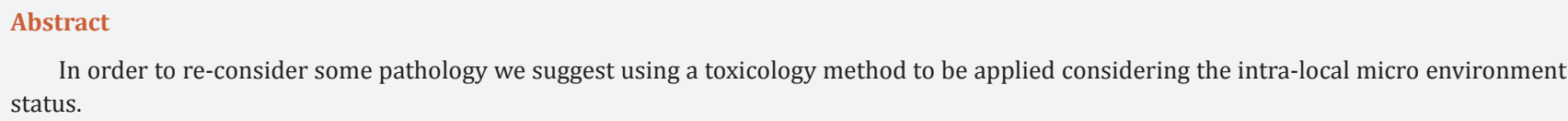

Keywords: Toxicology; Metabolic status; Unbalance; Gradients; Kinetics

\section{Introduction}

We start our paper observing that in past centuries physicians in some cardiologic disease were used to prescribe to the patient to take a long rest in order to recuperate their health status. In past centuries it wasn't available high efficient drugs strategies and so to take a long rest without physical and psychological stress contributes to this process.

Also in oriental medicine we can see body balances strategies, and also in some psycoaanalitc techniques we can see that time is relevant to re-equilibrate some conditions (in example acute stress). We can think that the main factors involved are TIME and LONG REST in order to balance the physiologic functions. In some case it was observed complete resolve in some situations. In example we can think that a metabolic unbalances can create this situation and the time make possible to restore.

In example what happen in sudden death heart syndrome in untrained? [1] Why in this condition physical training can reduce this event? We can think a condition of intra- toxicity time related. Other example can be some immune shock [2] or what happen in septic shock.

We think in this condition is relevant observe the time related intra- toxicity situation involved in some

Metabolic- catabolic -electrical cell membrane - immune status and other. In example involved in some heart aritmia, epilectic status, septic shock and other situation high time related (ischemic coronaric spasm etc). In embryology, oncology, toxicology, some heart and brain pathologies time is relevant added to local micro environment and inters cellular communication (crisis). We can consider an intra- local toxicology aspect time related to better verify some pathologic process under a new light.

According to $\mathrm{K}$ Michael Pollard et al. in example "Susceptibility to most autoimmune diseases is dependent on polygenic inheritance, environmental factors, and poorly defined stochastic events. One of the significant challenges facing autoimmune disease research is in identifying the specific events that trigger loss of tolerance and autoimmunity. Although many intrinsic factors, including age, sex, and genetics, contribute to autoimmunity, extrinsic factors such as drugs, chemicals, microbes, or other environmental factors can also act as important initiators. This review explores how certain extrinsic factors, namely drugs and chemicals, can promote the development of autoimmunity, focusing on a few better characterized agents that, in most instances, have been shown to produce autoimmune manifestations in human populations. Mechanisms of autoimmune disease induction are discussed in terms of research obtained using specific animal models. Although a number of different pathways have been delineated for drug/chemical-induced autoimmunity some similarities do exist and a working model is proposed [3]."

Blatt et al. [4] writed that: "Autism is defined neuro developmental disorder that affects over $1 \%$ of new births in the USA, about $2 \%$ of boys. The etiologies are unknown and they are genetically complex. There may be epigenetic effects, environmental influences, and other factors that contribute to the mechanisms and affected neural pathway. Involved brain specific areas in the cerebellum, limbic system, and cortex. Part 
of structures appears to be affected most rather than the entire structure, for example, select nuclei of the amygdala, the fusiform face area, and so forth. Altered cortical organization, frequent and narrower minicolumns and early overgrowth of the frontal portion of the brain, affects connectivity. Abnormalities include cytoarchitectonic laminar differences, excess white matter neurons, decreased numbers of GABAergic cerebellar Purkinje cells, and other events that can be traced developmentally and cause anomalies in circuitry. Problems in neurotransmission are evident according recent receptor/binding site studies especially in the inhibitory GABA system transmission likely contributing to an imbalance of the system of excitatory/ inhibitory transmission. As postmortem findings are related to core behavior symptoms, and technology improves, researchers are gaining a much better perspective of contributing factors to the disorder [4]".

According WEI $\mathrm{H}$. et al "the mechanisms responsible of autism pathogenesis are not understood, studies

have suggested that localized inflammation of the CNS may contribute to the development of autism. Recent evidence shows that IL- 6 has a crucial role in the development and plasticity of CNS [5] Katritsis et al. [6] write that "CAD is the predominant cause of SCD in older athletes.

Vigorous exertion can trigger cardiac arrest or SCD, especially in untrained persons, but habitual vigorous exercise diminishes the risk of sudden death during vigorous exertion [6]. "According the recent paper "Brain and Transmission Signal Modulation " 2017 "As observed in other scientific or medical discipline controlling some non physiological cellular activity Results in reducing of abnormal organ activation [7]".

According to Hung et al. [1] "Coronary artery spasm (CAS), an intense vasoconstriction of coronary arteries that causes total or subtotal vessel occlusion, plays an important role in myocardial ischemic syndromes including stable and unstable angina, acute myocardial infarction, and sudden cardiac death. Coronary angiography and provocative testing usually is required to establish a definitive diagnosis. While the mechanisms underlying the development of CAS are still poorly understood, CAS appears to be a multifactorial disease but is not associated with the traditional risk factors for coronary artery disease. The diagnosis of CAS has important therapeutic implications, as calcium antagonists, not $\beta$-blockers, are the cornerstone of medical treatment. The prognosis is generally considered benign; however, recurrent episodes of angina are frequently observed. We provide a review of the literature and summarize the current state of knowledge regarding the pathogenesis of CAS [8]."

And "Precipitating factors may contribute to the onset of CAS and act in the same patient to cause angina in different conditions. CAS can be precipitated by physical and/or mental stress 54, magnesium deficiency 55, alcohol consumption
56, the cold pressure test, hyperventilation, the Valsalva maneuver, remnant lipoproteins 57, and the administration of pharmacological agents such as cocaine 58 , sympathomimetic agents (epinephrine, norepinephrine, etc.), beta-blocking agents (propranolol, etc.), parasympathomimetic agents (methacholine, pilocarpine, etc.), and ergot alkaloids (ergonovine, ergotamine, etc.), particularly in the morning when spontaneous CAS is most likely to occur 26. Activated platelets may trigger CAS by releasing vasoconstrictor substances, including thromboxane and serotonin, both of which are found to be associated with CAS 59-61. It is important to differentiate CAS from Kounis syndrome because there is some overlap between the 2 entities. Kounis syndrome is characterized by the concurrence of acute coronary events with allergic or hypersensitivity reactions 62. To differentiate Kounis syndrome from CAS, knowledge of individual hypersensitivity is crucial [8]."

"Pathogenesis: The causes and the mechanisms underlying the development of CAS are still poorly defined and are likely multifactorial. In the 1980s, the autonomic nervous system was found to play an important role in the pathophysiology of CAS $27,63,64$. In the 1990s, inflammation, endothelial dysfunction, oxidative stress, respiratory alkalosis and magnesium deficiency were identified as predisposing factors 27. In the late 1990s and early 2000s, genetic mutations were found to be associated with CAS 27. Nonetheless, coronary vascular smooth muscle cell hyper reactivity seems to constitute the substrate for CAS [8]."

\section{Clas Linnman et al. writed that}

"Individual differences in ability to control fear have been linked to activation of dorsal anterior cingulate cortex, ventromedial prefrontal cortex, and amygdala. This study investigated whether functional variance in this network can be predicted by resting metabolism in these same regions.

Healthy subject volunteers were studied with positron emission tomography using [18F]-deoxyglucose to measure resting brain metabolism. This was followed by a two-day fear conditioning and extinction training paradigm in a functional magnetic resonance imaging scanner to measure brain activation during fear extinction and its recall. Skin conductance response was used to index conditioned responding. Resting metabolism in amygdala, dorsal anterior cingulate cortex and ventromedial prefrontal cortex were used to predict responses during fear extinction and extinction recall.

During extinction training, resting amygdala metabolism positively predicted ventromedial prefrontal cortex, and negatively predicted dorsal anterior cingulate cortex, activation. In contrast, during extinction recall, resting amygdala metabolism negatively predicted ventromedial prefrontal cortex, and positively predicted dorsal anterior cingulate cortex, activation. Resting dorsal anterior cingulate cortex metabolism predicted fear expression (skin conductance response) during extinction recall. Brain metabolism at rest predicts neuronal 
reactivity and skin conductance changes associated with recall of the fear extinction memory [9]."

\section{Discussion and Conclusion}

In toxicology usually are high considered the external environmental factors but we think we must observe under toxicological methods also the inside intra-extra cellular local microenvironment (paraphysiologic-pathologic conditions).

In some pathology the time is relevant added to local micro environment and inters cellular communication situations. We must consider an intra- local toxicology aspect time related to better verify some pathologic process under a new light.

In some time related local metabolic-catabolic-toxic status we can observe some cellular effect resulting in some organ failure. The time involved in resolve some temporary gradients or the velocity involved in this process can be fundamental.

The same effect related to too much rapid evolution or too slow reduction in balancing equilibrates physiologic systems. (The same the reduced effect showed in example in sports trained in SCD vs not trained activate platelet in trigger coronary artery spams, amygdala temporal iper activation or other examples).

We need to introduce more toxicological methods in some pathologies in order to clear some relevant aspect in etiology, diagnosis and therapy.

\section{Clarification}

This paper has not any diagnostic or therapeutic intent only to produce new research hypothesis.

\section{References}

1. Luisetto M, Luca C, Farhan A K, Ghulam RM (2017) Sudden Heart Pathology-a New Research Hipotesys. J Cardiol \& Cardiovasc Ther 8(1): 555730.

2. Luisetto M (2017) "Immune Shock - Chronologic Event in Some Brain Pathology". Current Opinions in Neurological Science 1(4): 207-215.

3. Pollard KM, Hultman P, Kono DH (2010) Toxicology of autoimmune diseases. Chem Res Toxicol 23(3): 455-466.

4. Gene J Blatt (2012) “The Neuropathology of Autism”. Scientifica 2012 (2012): Article ID 703675.

5. Wei H, Zou H, Sheikh AM, Malik M, Dobkin C, et al. (2011) IL-6 is increased in the cerebellum ofautistic brain and alters neural cell adhesion, migration and synaptic formation. J Neuroinflammation 8: 52.

6. Katritsis DG, Gersh BJ, Camm AJ (2016) A Clinical Perspective on Sudden Cardiac Death. Arrhythm Electrophysiol Rev 5(3): 177-182.

7. Luisetto M (2017) Short communication Brain and Transmission Signal Modulation theramostic brain disorder.

8. Hung MJ, Hu P, Hung MY (2014) Coronary artery spasm: review and update. Int J Med Sci 11(11): 1161-1171.

9. Linnman C, Zeidan MA, Furtak SC, Pitman RK, Quirk GJ, et al. (2012) Resting amygdala and medial prefrontal metabolism predicts functional activation of the fear extinction circuit. Am J Psychiatry 169(4): 415-423.

\section{Your next submission with Juniper Publishers will reach you the below assets}

- Quality Editorial service

- Swift Peer Review

- Reprints availability

- E-prints Service

- Manuscript Podcast for convenient understanding

- Global attainment for your research

- Manuscript accessibility in different formats

( Pdf, E-pub, Full Text, Audio)

- Unceasing customer service

Track the below URL for one-step submission https://juniperpublishers.com/online-submission.php 